Supporting information:

\title{
Preparation and Properties of Semi-Self-Assembled Lipopeptide
}

\section{Vesicles}

Dong Wang ${ }^{\dagger} *$, Bente $\mathrm{Ma}^{\dagger}$, Yurong Zhao ${ }^{\dagger}$, Yawei Sun ${ }^{\dagger}$, Yuxia Luan ${ }^{\ddagger}$, and Jiqian Wang $^{\dagger}, *$

${ }^{\dagger}$ State Key Laboratory of Heavy Oil Processing \& Centre for Bioengineering and Biotechnology, China University of Petroleum (East China), Qingdao, 266580, China

\$ School of Pharmaceutical Science, Key Laboratory of Chemical Biology (Ministry of Education), Shandong University, 44 West Wenhua Road, Jinan, Shandong, 250012, China

CORRESPONDING AUTHOR E-mail: jqwang@upc.edu.cn wangdong@ upc.edu.cn

Table: 1

Figures: 11

Pages: 7 


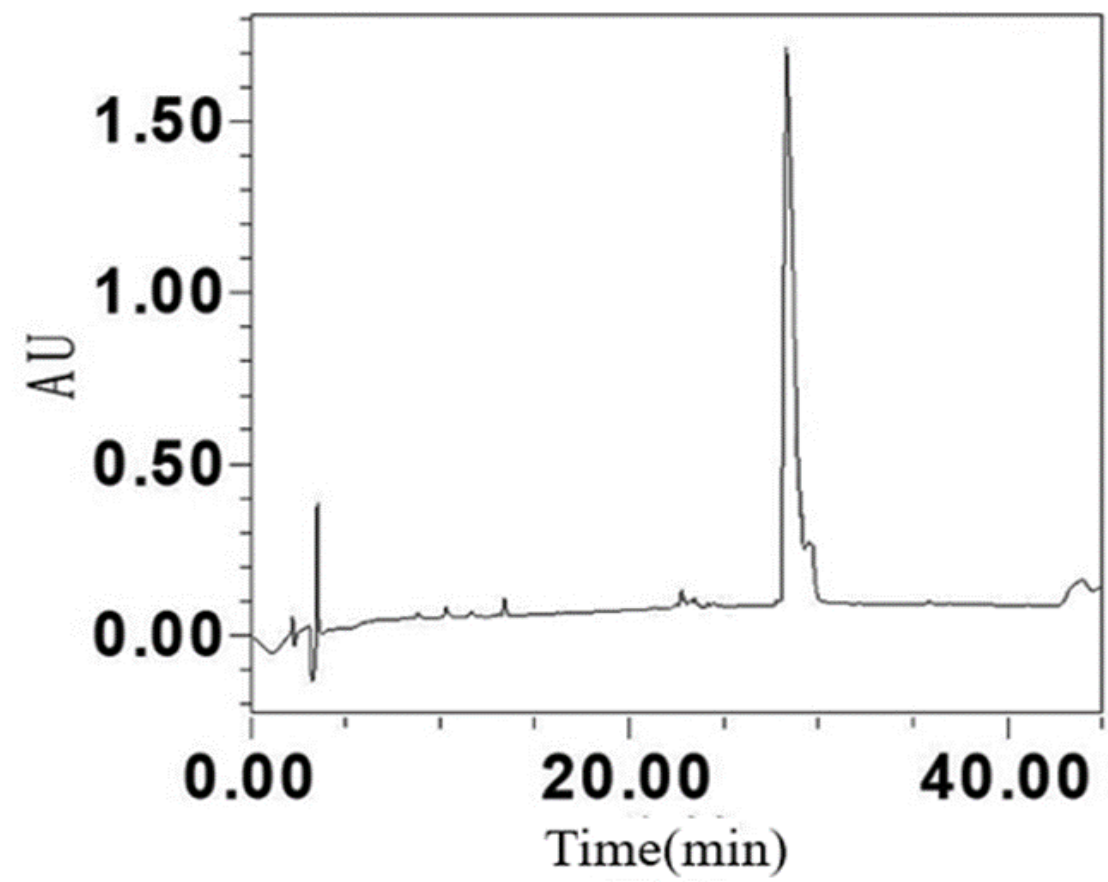

Figure S1.RP- HPLC of OASS

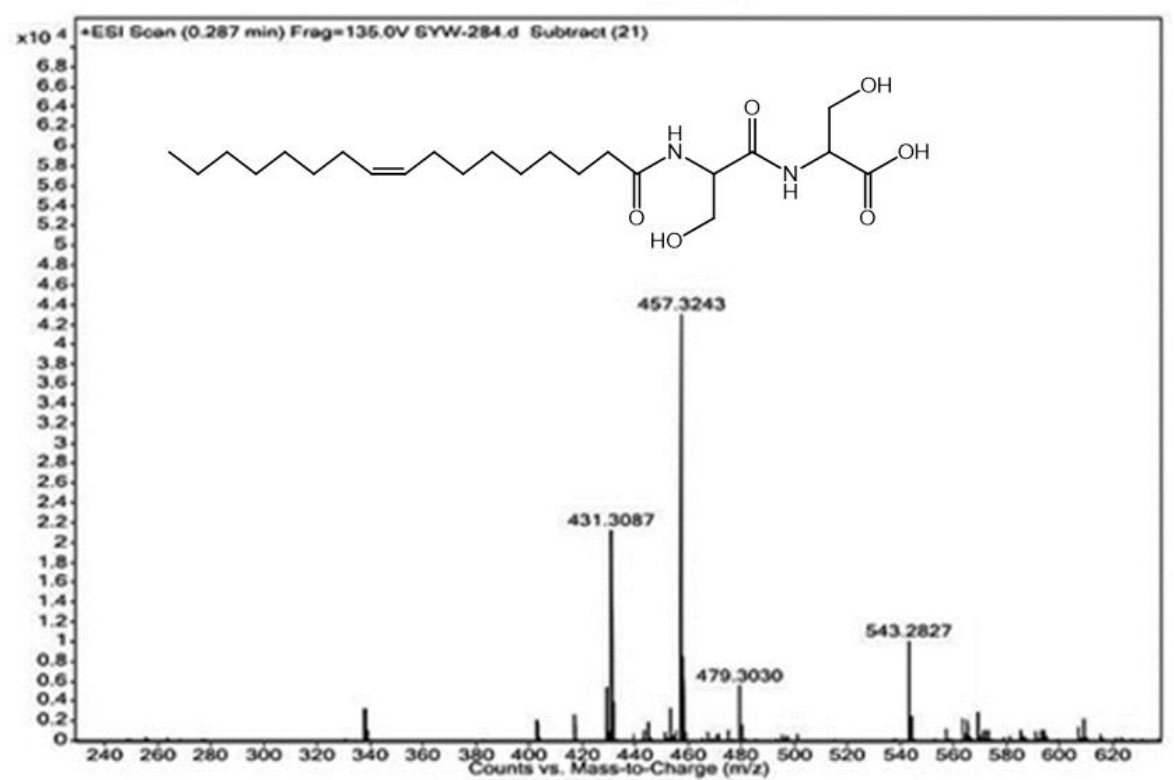

Figure S2. The MS spectrum of OASS. 
Table S1. Theoretical molecular mass of OA-SS and actual molecular mass analyzed by mass spectrometer

\begin{tabular}{cccc}
\hline & {$[\mathrm{M}+\mathrm{H}]^{+}$} & {$[\mathrm{M}+\mathrm{Na}]^{+}$} & {$[\mathrm{M}+\mathrm{K}]^{+}$} \\
\hline $\begin{array}{c}\text { Theoretical molecular } \\
\text { mass }\end{array}$ & 457.3 & 479.3 & 497.3 \\
Actual molecular mass & 457.3 & & \\
\hline
\end{tabular}

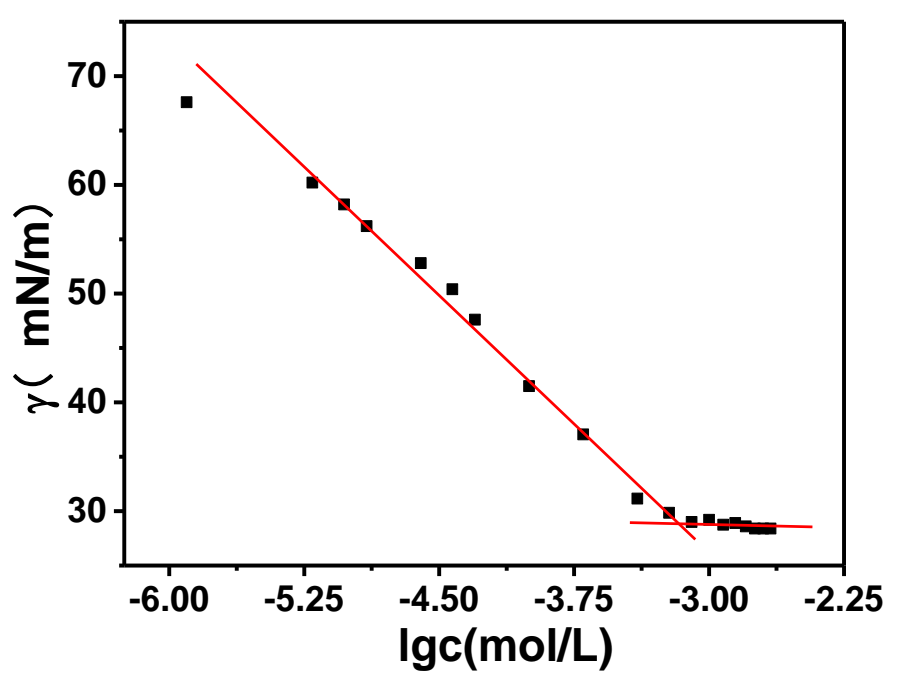

Figure S3. Surface tension measurement of OASS 


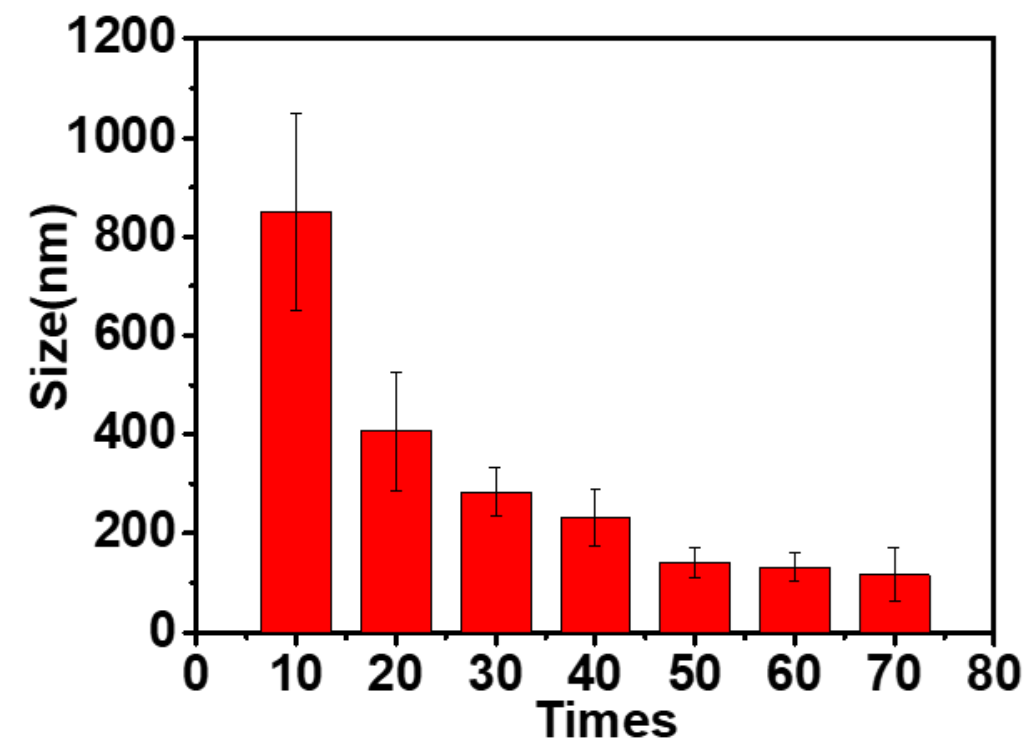

Figure S4. Vesicle size variation with extrusion times.

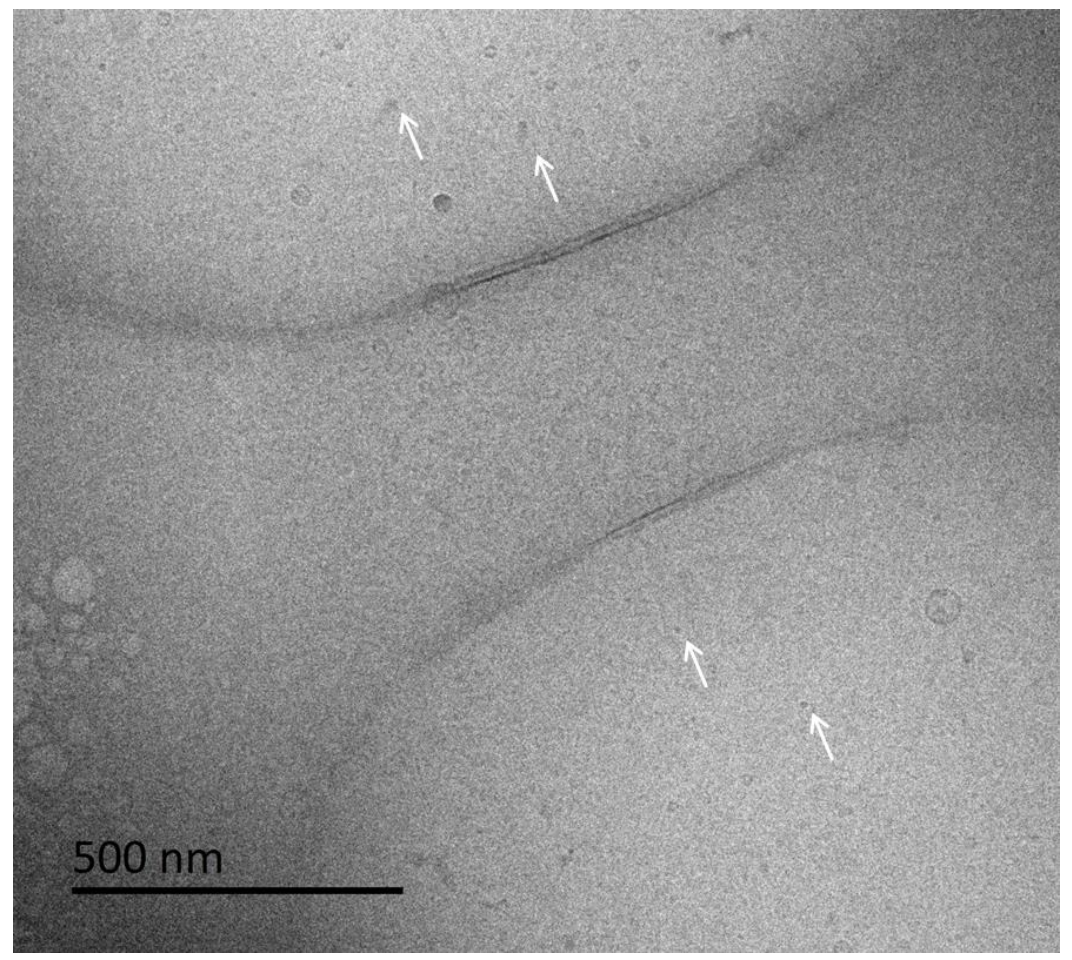

Figure S5. Cryo-TEM image of $4 \mathrm{mg} / \mathrm{ml} \mathrm{OASS}$ at $\mathrm{pH} 8.5$ 
Figure S6. Cryo-TEM image of $4 \mathrm{mg} / \mathrm{ml}$ OASS at $\mathrm{pH} 4.0$.
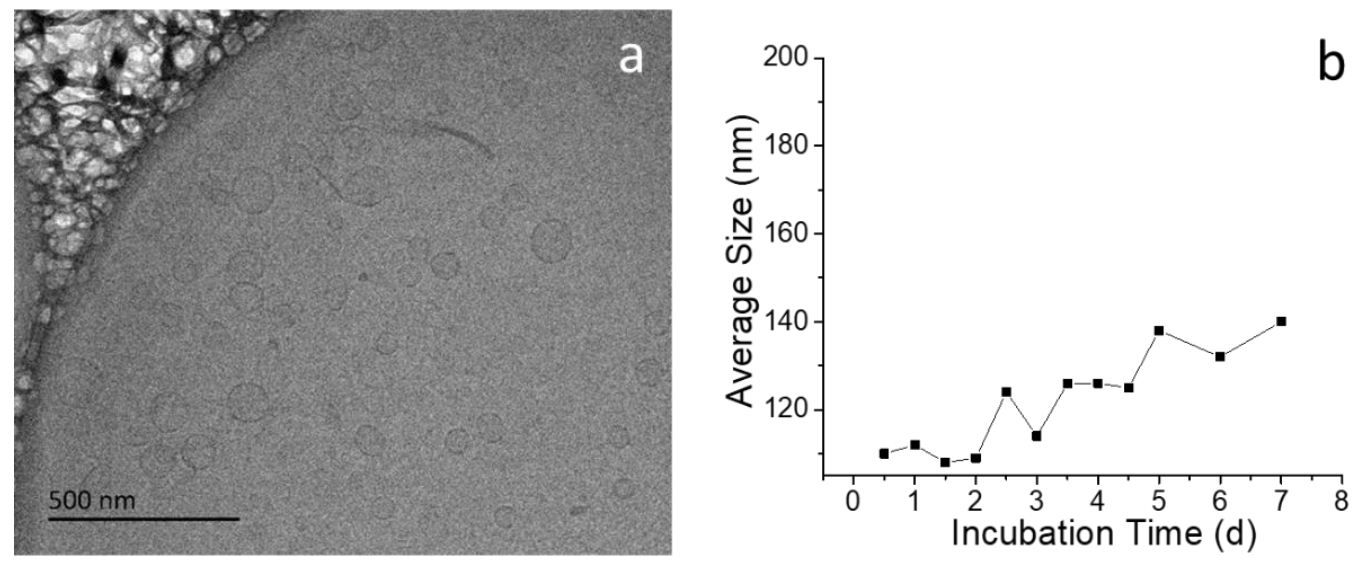

Figure S7. Cryo-TEM image of SSAL vesicles (a) and size stability (b) in PBS solutions at $\mathrm{pH} 7.4$. 


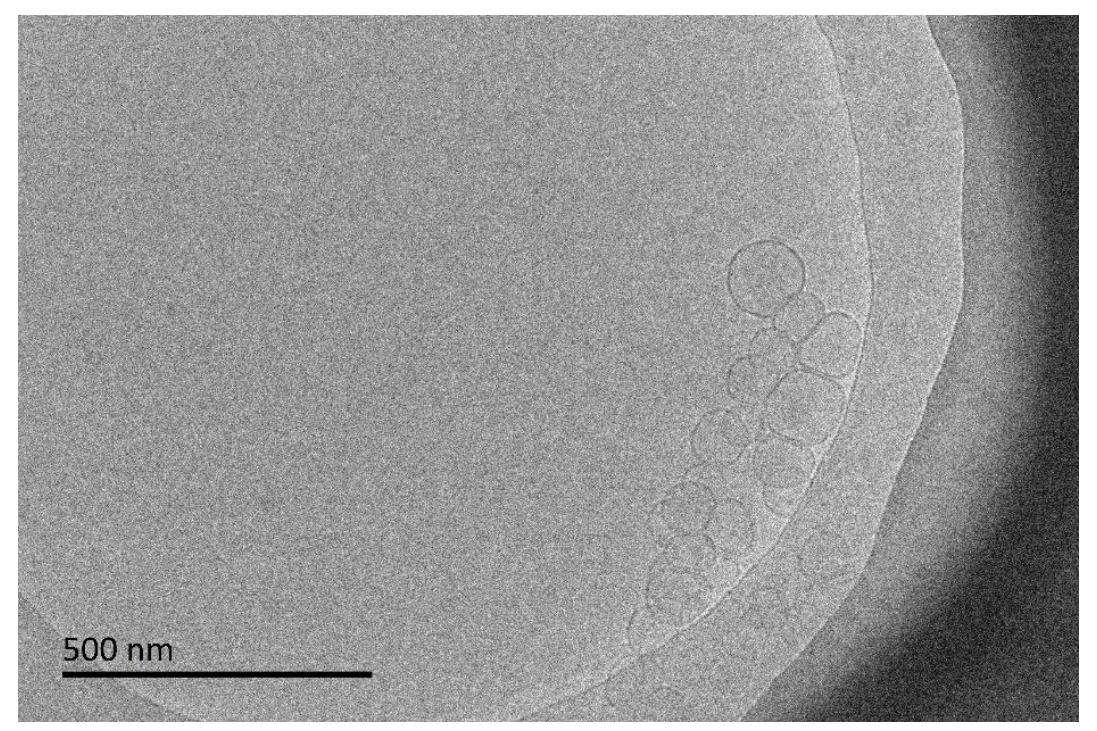

Figure S8. Cryo-TEM image of SSAL vesicles after freeze-dried storage.

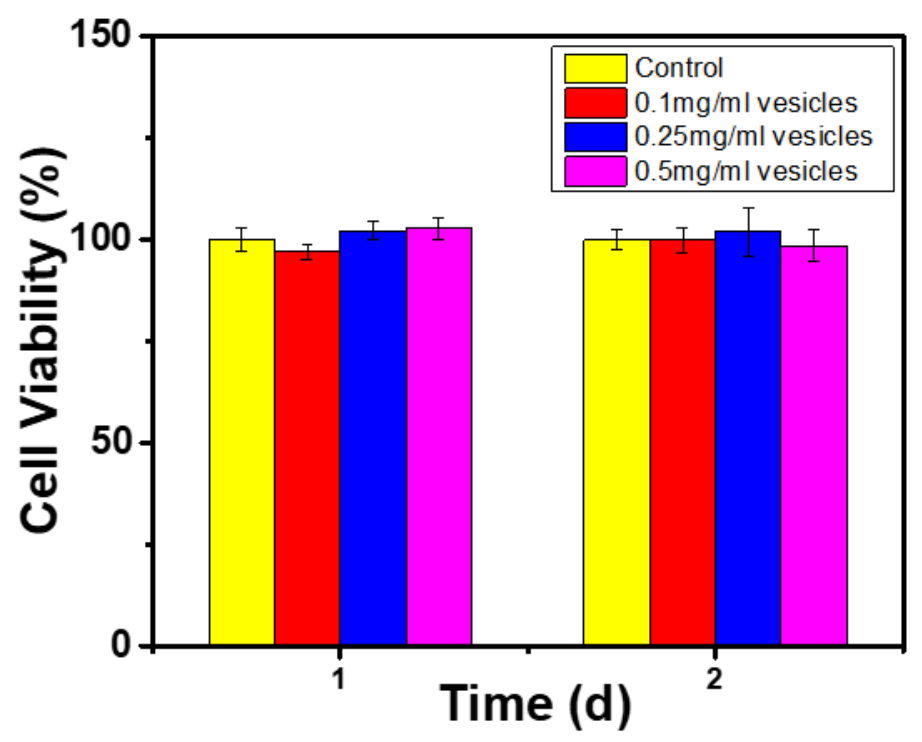

Figure S9. MTT assays of empty vesicles 

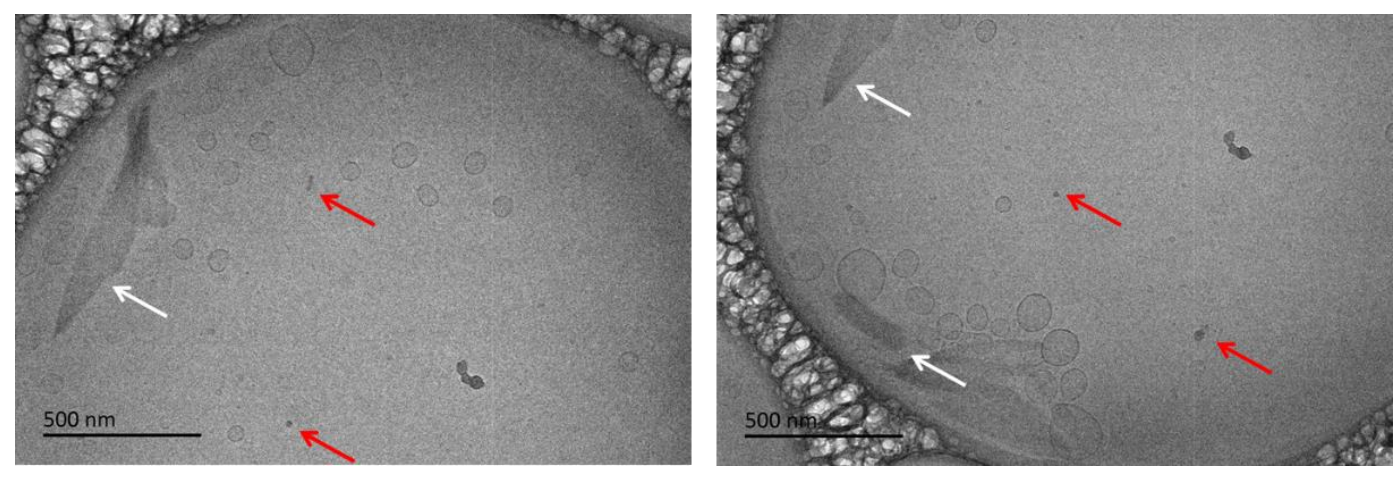

Figure S10. Cryo-TEM images of SSAL vesicles and protease in PBS solutions
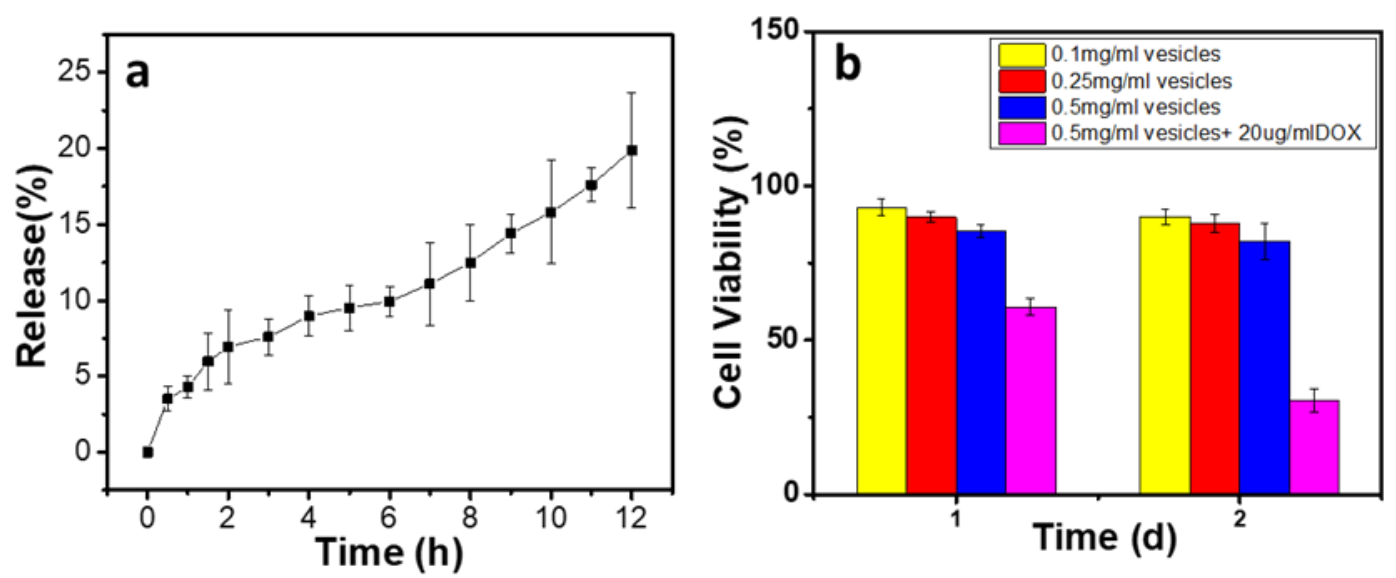

Figure S11. DOX $\cdot \mathrm{HCl}$ release behavior (a) and cytotoxicity (b) of CTAFe modified vesicles. 